\title{
Quimioprevención de neoplasias colorrectales en pacientes con neoplasias colónicas previas
}

\author{
Colorectal neoplasia chemoprevention in patients with previous colorectal neoplasia
}

\section{Objetivos}

Comparar la eficacia y la seguridad de distintos fármacos -aspirina, otros anti inflamatorios no esteroides (AINE)- y/o suplementos -calcio, vitamina $\mathrm{D}$, ácido fólico- en la prevención de neoplasias colorrectales metacrónicas avanzadas.

\section{Fuente de datos}

Se revisaron MEDLINE, Embase, Scopus, Web of science, Cochrane Central Register of Controlled Trials and Cochrane Database of Systematic Reviews desde 1999 hasta 2015.

\section{Selección de estudios}

Fueron seleccionados ensayos clínicos multicéntricos aleatorizados que hubieran incluido pacientes con antecedentes de neoplasias colorrectales en tratamientos con intención curativa antes de la aleatorización; y además hubieran sido asignados a alguna alternativa de quimioprevención o placebo, con el objetivo de prevenir la aparición de neoplasias metacrónicas.

Extracción, síntesis y análisis de datos

La eficacia fue evaluada a través de la incidencia de neoplasias
Dulai P y col. BMJ 2016; 355, i6188, doi 10.1136/bmj.i6188 colorrectales entre los tres y cinco años desde la colonoscopia índice; y la seguridad, mediante la determinación de efectos adversos serios (muerte, internación, trastornos vasculares, sangrados o suspensión del tratamiento por algún efecto adverso). Los autores realizaron un metaanálisis mediante una red bayesiana. La calidad de la evidencia fue evaluada con los criterios GRADE (en inglés, Grade of Recommendations Assessment, Development and Evaluation).

\section{Resultados}

Del total de los trabajos identificados fueron seleccionados 14 estudios que habían incluido 12.234 pacientes sometidos a diferentes estrategias de intervención. Como lo describe la tabla 1, la alternativa más eficaz para la prevención de aparición de neoplasias metacrónicas fue el uso de analgésicos no esteroideos (AINE) diferentes a la aspirina. Los suplementos de calcio, vitamina $D$ y/o ácido fólico no fueron eficaces, y tampoco pudo documentarse eficacia de ninguno de los tratamientos evaluados para la prevención de lesiones cancerosas.

Tabla 1. Riesgo de desarrollo de neoplasia metacrónica entre 3 y 5 años según la rama de tratamiento recibido

\begin{tabular}{l|c|c|c|c} 
& $\begin{array}{c}\text { Riesgo de neoplasia } \\
\text { metacrónica }\end{array}$ & $\begin{array}{c}\text { Eficacia } \\
\text { OR (IC95\%) }\end{array}$ & $\begin{array}{c}\text { Efectos adversos } \\
\text { OR (IC95\%) }\end{array}$ & Calidad de la evidencia \\
\hline Riesgo Control Asumido & $16,3 \%$ & - & - & - \\
\hline Aspirina & $12,1 \%$ & $0,71(0,41 \mathrm{a} 1,23)$ & $0,78(0,43$ a 1,38$)$ & Baja \\
\hline AINEa diferente de aspirina & $6,7 \%$ & $0,37(0,24 \mathrm{a} 053)$ & $1,23(0,95$ a 1,64$)$ & Alta \\
\hline
\end{tabular}

AINE: analgésico no esteroideo. Sulindac o celecoxib.

\section{Conclusión}

Entre los individuos que tuvieron una neoplasia colorrectal, los AINE diferentes de aspirina son los más eficientes en la prevención de neoplasias colorrectales avanzadas metacrónicas mien- tras que la aspirina en dosis bajas tiene el mejor perfil riesgobeneficio.

\section{Comentario}

Por un lado, la incidencia y la morbimortalidad del cáncer colorrectal, y por otro, su pasibilidad de ser reducidas a través de las intervenciones disponibles, justificarían el estudio de acciones de prevención en la población en riesgo. Si bien sus resultados no tuvieron significancia estadística, en el trabajo que acabamos de resumir, el uso de aspirina se asoció con una reducción del riesgo absoluto de desarrollar adenomas parecida a la comunicada por estudios previos que habían incluido menor cantidad de pacientes $(4,2 \% \text { vs. } 6,7 \%)^{1}$, lo que también también había sido comunicado por otros autores ${ }^{2,3}$, respecto de la prevención de la aparición de adenomas avanzados.

Desde 2015 la Fuerza de Tareas de Servicios Preventivos de EE.UU. (en inglés, United States Preventive Services Task Force) recomienda - grado B - el uso de aspirina en bajas dosis para la prevención de cáncer colorrectal en personas con una probabilidad a diez años de sufrir un evento cardiovascular mayor a $10 \%$, aprovechando el beneficio preventivo de su uso para ambas condiciones clínicas con el mismo riesgo de efectos adversos ${ }^{4}$. Vale destacar que una revisión sistemática con meta-análisis en red ${ }^{5}$, ya había documentado que el efecto beneficioso de la aspirina sobre la mortalidad por cáncer colorrectal es similar al del rastreo con recto-sigmoideoscopía, y superior a este respecto de la mortalidad por cáncer de colon proximal.

\section{Conclusiones del comentador}

El perfil de riesgo de efectos adversos no respalda el uso quimiopreventivo en forma rutinaria de los AINE diferentes a aspirina. EI uso de aspirina podría ser considerado para la prevención de adenomas avanzados y adenocarcinomas, sin que pueda todavía precisarse la duración del tratamiento, la población que puede beneficiarse, ni tampoco la magnitud del efecto protector luego de cesada su administración.

Ricardo Esteban Mentz [ Servicio de Cirugía General del Hospital Italiano de Buenos Aires. ricardo.mentz@ hospitalitaliano.org.ar ]

Mentz R. Quimioprevención de neoplasias colorrectales en pacientes con neoplasias colónicas previas. Evid Act Pract Ambul. 2017:20(3):Jul-Sep-66. Comentado de: Dulai PS. Chemoprevention of colorectal cancer in individuals with previous colorectal neoplasia: systematic review and network meta-analysis. BMJ. 2016;355:i6188. PMID 27919915.

\section{Referencias bibliográficas}

1. Cole BF y col. Aspirin for the chemoprevention of colorectal adenomas: meta-analysis of the randomized trials. J Natl Cancer Inst. 2009; 101(4): 256-66.

2. Gao F y col. The effect of aspirin in the recurrence of colorectal adenomas: a meta-analysis of randomized controlled trials. Colorectal Dis. 2009; 11(9): $893-901$.

3. Baron JA y col. A randomized trial of aspirin to prevent colorectal adenomas. N Engl J Med. 2003; 348(10):891-9.

4. Bibbins-Domingo K; U.S. Preventive Services Task Force. Aspirin Use for the Primary Prevention of Cardiovascular Disease and Colorectal Cancer: U.S. Preventive Services Task Force Recommendation Statement. Ann Intern Med. 2016 Jun 21;164(12):836-45. doi: 10.7326/M16-0577. Epub 2016 Apr 12.

5 . Emilsson $L$ y col. Systematic review with meta-analysis: the comparative effectiveness of aspirin vs. screening for colorectal cancer prevention. Aliment Pharmacol Ther. 2017;45(2):193-204. 